\title{
LABORATORIO DE INMUNOENDOCRINOLOGÍA
}

\section{LABORATORY OF IMMUNOENDOCRINOLOGY}

El Laboratorio de Inmunoendocrinología (LIE) funciona en la Cátedra de Inmunología de la Facultad de Farmacia y Bioquímica (FFyB) de la Universidad de Buenos Aires (UBA) e Instituto de Estudios de la Inmunidad Humoral Prof. Ricardo A Margni (IDEHU), CONICET-UBA.

El líder natural del LIE es el Dr. Edgardo Poskus, quien actualmente se desempeña como Profesor Consulto de la UBA e Investigador Contratado del CONICET. Además el laboratorio lo integran los siguientes profesionales:

- Dra. Silvina N. Valdez: investigadora independiente del CONICET, Directora del Laboratorio de Prestación de Servicios desde mayo de 2014.

- Dra. Aldana Trabucchi: investigadora asistente del CONICET.

- Dra. Silvina Bombicino: becaria posdoctoral ANPCYT.

- Dr. Rubén F. lacono: profesional principal de la Carrera de Personal de Apoyo a la Investigación Científica del CONICET.

- Dr. Alberto Penas Steinhardt: profesional asistente de la Carrera de Personal de Apoyo a la Investigación Científica del CONICET.

- Bioq. Luciano Guerra: tesista de la UBA.

- Bioq. y Farm. Natalia I. Faccinetti: tesista de la UBA.

- Est. de Bioq. Adriana V. Sabljic: becaria estímulo de la UBA.

- Est. de Farm. Bruno D. Rovitto: becario estímulo de la UBA.

- Est. de Bioq. Juan Ignacio Marfía.

Desde hace años nuestro laboratorio ha desarrollado una actividad original en el tema de la inmunología de la diabetes mellitus (DM). En particular se ha enfocado en el estudio de las variantes de esa patología
(DM autoinmune -DMA-) en las que el componente inmunológico autoagresivo integra las bases etiopatogénicas y donde puede demostrarse serológicamente su existencia temprana y también puede contribuirse al apoyo diagnóstico preciso una vez que la enfermedad se ha expresado clínicamente. De este modo, el laboratorio se ha dedicado a la detección y caracterización de las formas de diabetes mediadas por autoinmunidad, tanto de comienzo en edad infanto-juvenil como en el adulto y adulto mayor. Resultados interesantes en esta área fueron publicados en las revistas especializadas Autoimmunity (2001, 2008, 2012), Medicina (2001), Revista de la SAD (2001, 2015), PLoS ONE (2012, 2013), European Journal of Endocrinology (2016) y Rev Argent Endocrinol Metab (2016).

Todos los integrantes del laboratorio realizan actividades de docencia e investigación. En este sentido, el laboratorio está conformado por investigadores, becarios que realizan sus tesis doctorales y algunos alumnos que comienzan con becas estímulo de la UBA. El foco del equipo de investigación está particularmente en el desarrollo de proteínas recombinantes (Papouchado y col., 1997; Santos y col., 2000; Trabucchi y col., 2012; Guerra y col., 2016) con el fin de aplicarlas en novedosos ensayos para la detección sensible de los autoanticuerpos (marcadores) de DMA (Papouchado y col., 1997; Valdez y col., 2003; Valdez y col., 2004; Valdez y col., 2004; Villalba y col., 2007; Valdez y col., 2007; Guerra y col., 2014). Nuestra idea es lograr un kit nacional de bajo costo que pueda comercializarse en laboratorios de mediana complejidad, incluso en zonas periféricas del interior del país.

El grupo ha recibido más de 40 subsidios 
de organismos públicos, entidades científicas y empresas desde 1985; desde 1983 ha publicado hasta el presente 36 trabajos en revistas extranjeras indexadas; obtuvo patentes en EE.UU. y Argentina sobre autoantígenos recombinantes relacionados a la DMA, incluyendo reivindicaciones sobre procedimientos analíticos originales que los aplican y se ha inscripto como laboratorio prestador de servicios analíticos oficiales.

Desde el año 1994 el LIE presta servicios analíticos de alta complejidad y está inscripto en la Subsecretaría de Transferencia Tecnológica de la Facultad de Farmacia y Bioquímica (UBA), especialidad: Inmunoanalítica Diabetológica (marcadores de autoinmunidad y test de seguimiento y control). En el año 2004 fue designado Laboratorio de Referencia y Servicio, Facultad de Farmacia y Bioquímica, UBA, Resol. CD 671, 27/04/04, y en el año 2005 fue autorizado como laboratorio prestador de servicios de alta tecnología (STAN) en el Instituto de Estudios de la Inmunidad Humoral Prof. Ricardo A. Margni (IDEHU, CONICET-UBA), Resol. No 2.370 del Directorio CONICET (26/12/05).

Los análisis especializados sirvieron para el apoyo diagnóstico discriminativo de la DMA en nuestro medio. EI LIE presta servicios de apoyo diagnóstico con la determinación de marcadores humorales de autoinmunidad anticélulas beta de los islotes pancreáticos y sus productos: IAA/PAA, GADA, IA-2A y ZnT8A. Los parámetros analíticos de los métodos desarrollados se someten a controles de calidad periódicos en medios internacionales.

En la actualidad nuestro laboratorio se está expandiendo hacia otras enfermedades autoinmunes, las cuales comparten alelos de susceptibilidad con la DMA y se presentan en simultaneidad en alto porcentaje de los pacientes. Éstas son la enfermedad tiroidea autoinmune (ETA) y la enfermedad celíaca (EC). EI LIE se encuentra abocado al desarrollo de una plataforma biotecnológica para la síntesis de los distintos autoantígenos recombinantes involucrados en las tres patologías autoinmunes para aplicarlos en novedosos inmunoen- sayos multiplex y en la detección simultánea de los autoanticuerpos.

Las principales publicaciones del grupo de investigación son:

- Papouchado ML, Valdez SN, GhiringheIli D, Poskus E, Ermácora MR. Expression of properly-folded human glutamic acid decarboxylase 65 as a fusion protein in Escherichia coli. Eur J Biochem 1997; 246, 350-359.

- Papouchado ML, Valdez SN, Ermácora MR, Gañan S, Poskus E. Highly-sensitive and specific enzyme-linked immunosorbent assays for GAD65 autoantibodies using a thioredoxin-GAD65 fusion antigen. J Immunol Methods 1997; 207, 169-178.

- Santos J, Antón E, Marino Busjle C, Valdez SN, Villanueva A, Sica M, lacono R, Maffia P, Poskus E, Ermácora M. Replacement of methionine 161 with threonine eliminates a major by-product of human glutamic acid decarboxylase 65 expression in E. coli. Biotechnol Appl Biochem 2000; 31, 205-212.

- Valdez SN, Sica M, Labovsky V, lacono R, Cardoso A, Krochik G, Mazza CS, Ermácora M, Cédola N, Poskus E. Combined measurement of diabetes mellitus immunological markers: an assessment of its benefits in adult-onset patients. Autoimmunity 2001; 33, 227-236.

- Krochik AG, Mazza CS, Valdez SN, Stumpo RR, Papouchado ML, lacono RF, Cardoso Landaburu AC, Sica MP, Ozuna B, Poskus E. Immunologic and genetic markers in insulin-dependent diabetes mellitus (type 1) in an Argentine population. Medicina 2001; 61, 279-283.

- Mazza CS, Krochik AG, Valdez SN, Stumpo RR, Papouchado ML, lacono RF, Cardoso Landaburu AC, Sica MP, Ozuna B, Poskus E. Marcadores inmunológicos y genéticos en diabetes mellitus tipo 1 en Argentina. Revista de la Sociedad Argentina de Diabetes 2001; 35 (3): 112-120.

- Valdez SN, lacono R, Villalba A, Cardoso A, Ermácora M, Poskus E. A radioligand-binding assay for detecting antibodies specific for proinsulin and insulin using 35S-proinsulin. Journal of Immunological Methods 2003; 279: 173-181. 
- Valdez SN, Villalba A, lacono R, Poskus E. Preparation of recombinant human 35S-proinsulin and its application in a combined radioligand-binding assay. Usefulness of the novel method as first-line screening for predicting insulin requirement in adult onset diabetic patients. Acta Bioquímica Clínica Latinoamericana 2004; 38 (1): 3-15.

- Valdez SN, Villalba A, lacono R, Poskus E. Single tube test for autoantibodies to glutamic acid decarboxylase and proinsulin as first-line screening for autoimmunity in adult onset diabetic patients. Clinical Immunology 2004; 113 (3): 241-247.

- Valdez SN, Villalba A, lacono R, Poskus E. Obtención de un trazador 35S-proinsulina y su aplicación en un ensayo radiométrico combinado para predecir el requerimiento de insulina en pacientes diabéticos adultos. Artículo publicado en la Sección Expertos Invitados de la Sociedad Iberoamericana de Información Científica 2005. Disponible en: www. siicsalud.com.

- Villalba A, Valdez SN, lacono R, Poskus E. Development of two alternative enzyme-linked immunosorbent assays for routine screening of glutamic acid decarboxylase autoantibodies. Clinica Chimica Acta 2007; 376: 82-87.

- Valdez SN, Villalba A, lacono R, Poskus E. Desarrollo e implementación de métodos inmunoanalíticos de baja complejidad para la detección precoz y el apoyo diagnóstico de la diabetes mellitus autoinmune. Revista Farmacéutica 2007; 149 (1): 42-56.

- Villalba A, lacono R, Valdez SN, Poskus E. Detection and immunochemical characterization of glutamic acid decarboxylase autoantibodies in patients with autoimmune diabetes mellitus. Autoimmunity 2008; 41 (2): 143-153.

- Carbajal ML, Soto Espinoza SL, Valdez SN, Poskus E, Smolko EE, Grasselli M. Arsenical grafted membranes for immobilization of thioredoxin-like proteins. Reactive \& Functional Polymers 2009; 69, 816-820.

- Valdez SN, Poskus E. Autoimmune diabetes mellitus: the importance of autoantibodies for disease prediction and diagnostic support. Current Immunology Reviews 2010; 6, 299-313.

- Trabucchi A, Faccinetti N, Guerra L, Puchulu F, Frechtel G, Poskus E, Valdez SN. Detection and characterization of ZnT8 autoantibodies could help to screen latent autoimmune diabetes in adult-onset patients with type 2 phenotype. Autoimmunity 2012; 45 (2), 137-142.

- Trabucchi A, Guerra L, Faccinetti N, lacono R, Poskus E, Valdez SN. Expression and characterization of human proinsulin fused to thioredoxin in Escherichia coli. Applied Microbiology and Biotechnology 2012; 94:1565-1576.

- Trabucchi A, Guerra L, Faccinetti N, lacono $R$, Poskus E, Valdez SN. Surface plasmon resonance reveals a different pattern of proinsulin autoantibodies concentration and affinity in diabetic patients. PLoS ONE 2012; 7(3): e33574. doi:10.1371/journal.pone.0033574

- Prieto M, Krochik AG, Chaler E, Maceiras M, Villalba A, Valdez SN, Cerrone G, Tellechea M, Mazza CS. Obesidad y factores de riesgo del síndrome metabólico en jóvenes con diabetes tipo 1. Medicina 2012; 72: 291-297.

- Trabucchi A, lacono R, Guerra L, Faccinetti N, Krochik AG, Arriazu M, Poskus E, Valdez SN. Characterization of insulin antibodies by surface plasmon resonance in two clinical cases: brittle diabetes and insulin autoimmune syndrome. PLoS ONE 2013; 8(12): e84099. doi:10.1371/ journal.pone.0084099.

- Guerra L, Trabucchi A, Faccinetti N, lacono R, Ureta D, Poskus E, Valdez SN. Flow cytometric microsphere-based immunoassay as a novel non-radiometric method for the detection of glutamic acid decarboxylase autoantibodies in type 1 diabetes mellitus. Analyst 2014; 139 (12): 3017-25.

- Ridner E, Yohena S, Tornelli FA, Muller C, Díaz S, Lovecchio S, Faccinetti NI, Penas Steinhard A, Valdez SN, Guerra L, Frechtel G. Autoinmunidad contra la célula beta en el adulto mayor con diabetes tipo 2: impacto clínico, metabólico y terapéutico. Revista de la Sociedad Argentina de Diabetes 2015; 49 (1): 5-12.

- Faccinetti N, Guerra L, Penas Steinhardt 
A, lacono R, Frechtel G, Trifone L, Poskus E, Trabucchi A, Valdez SN. Characterization of zinc transporter 8 antibodies in autoimmune diabetic patients from Argentinian population using monomeric, homodimeric and heterodimeric ZnT8 antigen variants. European Journal of Endocrinology 2016; 174 (2): 157-65.

- Sosa L, Torkko JM, Primo ME, Llovera RE, Ríos AS, González Flecha FL, Trabucchi A, Valdez SN, Poskus E, Solimena M, Ermácora MR. Biochemical, biophysical, and functional properties of ICA512/IA-2 RESP18 homology domain. Biochimica et Biophysica Acta 2016; 1864 511-522.

- Faccinetti NI, Rovitto BD, Guerra L, Sabljic AV, lacono RF, Trifone L, Poskus E, Trabucchi A, Valdez SN. Detección y caracterización de autoanticuerpos anti-ZnT8 en pacientes argentinos con diabetes mellitus tipo 1. Rev Argent Endocrinol Metab 2016; 53 (3): 77-83.

- Guerra L, Faccinetti NI, Trabucchi A, Rovitto BD, Sabljic AV, Poskus E, lacono RF, Valdez SN. Novel prokaryotic expression of thioredoxin-fused insulinoma associated protein tyrosine phosphatase 2 (IA-2), its characterization and immunodiagnostic application. BMC Biotechnology 2016; 16:84 doi:10.1186/ s12896-016-0309-2.

\section{Capítulos de libros}

- Cerrone G, Valdez SN, Poskus E, Frechtel G. Bases inmunogenéticas de la diabetes autoinmune: aplicación a la fisiopatología, clínica y tratamiento. En: Fisiopatología molecular y clínica endocrinológica (Eds. Calandra RS, Barontini MB) 2015; Neuhaus, Buenos Aires, Argentina, 1117-1130. 